\title{
Effectively Using the QRFM to Model Truck Trips in Medium-Sized Urban Communities
}

\author{
Michael D. Anderson ${ }^{1}$, Mary C. Dondapati ${ }^{1}$, Gregory A. Harris ${ }^{2}$ \\ ${ }^{1}$ Department of Civil and Environmental Engineering, University of Alabama in Huntsville, Huntsville, USA \\ ${ }^{2}$ Center for Management and Economic Research, University of Alabama in Huntsville, Huntsville, USA \\ Email: andersmd@uah.edu
}

Received March 21, 2013; revised April 22, 2013; accepted April 30, 2013

Copyright (C) 2013 Michael D. Anderson et al. This is an open access article distributed under the Creative Commons Attribution License, which permits unrestricted use, distribution, and reproduction in any medium, provided the original work is properly cited.

\begin{abstract}
This paper analyses the effectiveness of applying the Quick Response Freight Manual (QRFM) to model freight transportation. Typically, freight transportation is indirectly modeled or as an after-thought. Increasing freight volumes, coupled with cost saving strategies such as just-in-time delivery systems, require that transportation policymakers analyze infrastructure needs and make investment decisions that explicitly include freight volumes as a component. This paper contains a case study using a medium sized urban area travel model and the QRFM trip generation and a distribution methodology to provide a framework for freight planning that can be used to improve resource allocation decisions.
\end{abstract}

Keywords: Freight Modeling; Travel Demand Modeling

\section{Introduction}

The efficient and effective movement of freight is a critical component in the transformation and growth of the economy. Often, transportation planners use urban transportation planning models, which are representations of the existing transportation infrastructure to determine the impacts of future changes. These planning models are developed and validated to reflect existing traffic volumes and patterns. After validation, the models are used for forecasting daily traffic volumes on primary arterials and freeways and evaluate changes in roadway infrastructure and socio-economic characteristics. In small and medium sized urban communities, proper roadway infrastructure resource allocation decisions based on data obtained from the community's travel demand model and long-range transportation planning process could potentially be the determining factor between continued community growth and stagnation.

Since the modeling process is important, it is critical that the models used provide the best forecasts of future conditions. Unfortunately, freight transportation requirements are often not included in travel demand models developed and maintained in small communities, or else, freight trips are included in these models through very simplified methodologies.

This paper examines the potential to use available freight trip generation factors and a distribution scheme to determine freight transportation demand appropriate for incorporation into a community travel demand model. First, the paper presents background into travel demand forecasting and the Quick Response Freight Manual (QRFM) trip generation equations $[1,2]$. Next, the paper applies the model through a case study of Huntsville, AL, a medium-sized community in the north-central portion of the state. A statistical analysis of the QRFM technique is applied to the network using a variety of distribution schemes to improve its forecasting ability. The paper concludes that the proper incorporation of freight transportation needs into the travel demand modeling process can improve its results and should lead to improved investment decisions for the community.

\section{Transportation Planning Background and Freight Specifics}

The background for this paper is the traditional four step modeling process used in most small and medium sized urban areas and specifics of the process that deal with freight. The traditional transportation planning process follows the sequential four-step methodology: trip generation, trip distribution, mode split, and traffic assignment. The first step in the process, trip generation, uses socio-economic data, aggregated to traffic analysis zones, to determine the number of trips produced by and attracted to each zone in the study area [3]. For passenger 
transportation, factors that can influence trips produced from or attracted to a zone are: household income and size, automobile ownership, type of businesses, and trip purpose [3]. The trip generation step then converts these zonal data values into trip purposes. However, in most small and medium sized urban communities, there is no model developed for freight productions or attractions since it is time consuming and costly to survey businesses and manufacturers on their specific freight requirements.

Trip distribution connects trip origins and destinations for the development of a trip interchange matrix. The two main factors considered are trip length and the travel direction or orientation. The most common method used for trip distribution is a gravity model, which is based on Newton's law [3]. The gravity model predicts that trip interchanges between zones are directly proportional to the productions and attractions in the zones and inversely proportional to the spatial separation between zones [3]. In other words, zones with more activities or businesses are more likely to exchange more trips, and zones with longer distances between them are likely to exchange fewer trips. For freight, it is expected that the trip distribution would be similarly performed.

Modal split is used to estimate how many trips will use public transit and how many trips will use private vehicles, typically using a logit model [3]. However, this step of the process is generally ignored in small and medium sized communities, as transit ridership is not significant. With freight however, this step would contrast truck versus alternative modes of shipment (rail, water, and air) and therefore it is significant. As limited availability for alternate freight shipping models often exists in medium sized communities, this step is still not included in the modeling process.

Traffic from the modal split analysis is then assigned to available roadways or transit routes using Waldrop's equilibrium theorem, or some approximation of equilibrium. By this theory, under equilibrium conditions traffic arranges itself in congested networks in such a way that no individual trip maker can reduce his path costs by switching routes [3]. Regarding freight, it is not necessarily logical to assume freight shipments will likely change their route due to congestion effects, at least not off the major roadways within the communities.

To overcome the absence of freight in transportation models, the original Quick Response Freight Manual (QRFM) and its updated version QRFM II, were prepared for the Federal Highway Administration [1,2]. The objective of the reports were to provide background information on the freight transportation system and factors affecting freight demand to planners who may be relatively new to the inclusion of freight planning and to provide simple techniques and transferable parameters that can be used to develop commercial vehicle trip tables which can then be merged with passenger vehicle trip tables developed through the conventional four-step planning process. The QRFM report identifies trip generation factors that define production and attraction values manageable within a small community. To support trip distribution, the QRFM provides a series of friction factors that can be incorporated into the gravity model to specify the expected length of freight movements. Figure 1 provides the trip generation equations and Figure 2 the friction factor equations.

\section{Case Study: Huntsville, AL}

Huntsville, Alabama with a population of approximately 300,000 was selected as case study to analyze the incorporation of freight into the modeling process. For this research, the data on the transportation network for the City of Huntsville was acquired from the Huntsville Metropolitan Planning Organization (MPO); see Figure 3 [4].

The research was performed by applying the trip generation rates obtained from the QRFM to the socio-economic data collected by the Huntsville MPO. For each zone, the socio-economic data were converted into freight trips using the rates provided by the QRFM. A

\begin{tabular}{|c|c|c|c|c|}
\hline \multirow{2}{*}{ Generator } & \multicolumn{4}{|c|}{ Commercial Vehicle Trip Destinations (or Origins) per Unit per Day } \\
\hline & Four-Tire Vehicles & Single Unit Trucks (6 + Tires) & Combinations & TOTAL \\
\hline \multicolumn{5}{|l|}{ Employment } \\
\hline - Agriculture, Mining and Construction & 1.110 & 0.289 & 0.174 & 1.573 \\
\hline $\begin{array}{l}\text { - Manufacturing, Transportation, Communications, } \\
\text { Utilities and Wholesale Trade }\end{array}$ & 0.938 & 0.242 & 0.104 & 1.284 \\
\hline - $\quad$ Retail Trade & 0.888 & 0.253 & 0.085 & 1.206 \\
\hline - Office and Services & 0.437 & 0.068 & 0.009 & 0.514 \\
\hline - Households & 0.251 & 0.099 & 0.028 & 0.388 \\
\hline
\end{tabular}

Figure 1. Trip generation rates from the QRFM [2]. 


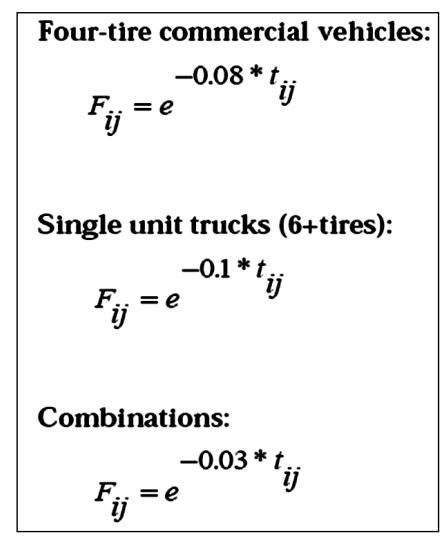

Figure 2. Friction factors from the original QRFM. (Source Cambridge Systematics, Inc. Quick Response Freight Manual II. Federal Highway Administration. Publication No. FHWA-HOP-08-010. September 2007).

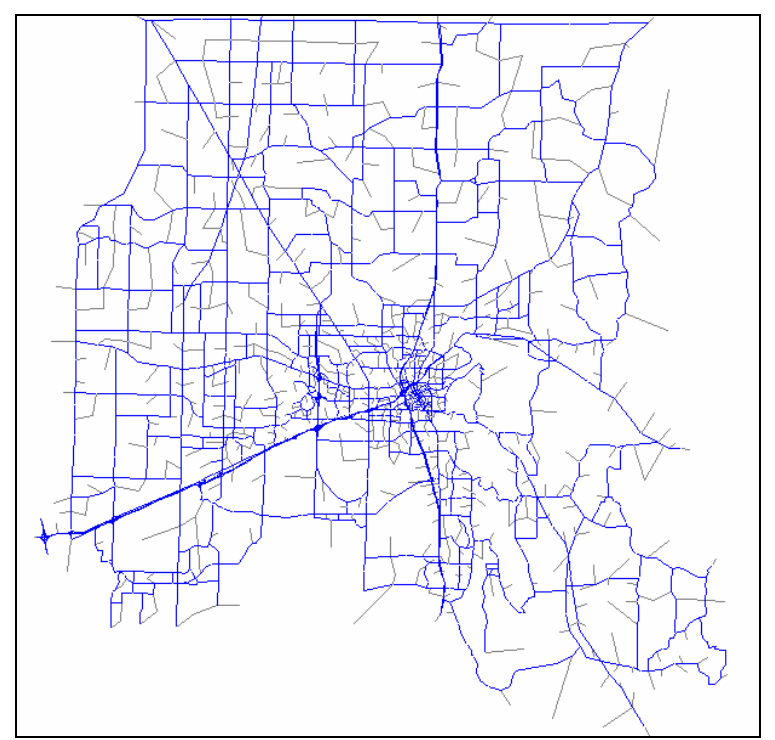

Figure 3. Huntsville, AL planning model.

visual validation of the trip generation model results as they related to the total non-retail employment in the study city was performed by developing a thematic map showing the amount of non-retail employment within each traffic analysis zone overlaid with a dot density plot of the freight trips (see Figure 4). The figure indicated that the QRFM freight trips were located in areas of higher non-retail employment. This result was expected and validated the use of the QRFM model in the planning process.

The Huntsville model was based on trip generation, distribution and assignment. Common with the practice in planning studies this model used the static traffic assignment technique. The rationale is that it mirrored the current modeling system used in the community and approved by the state for transportation forecasting processes. This ensured that the model would be accepted

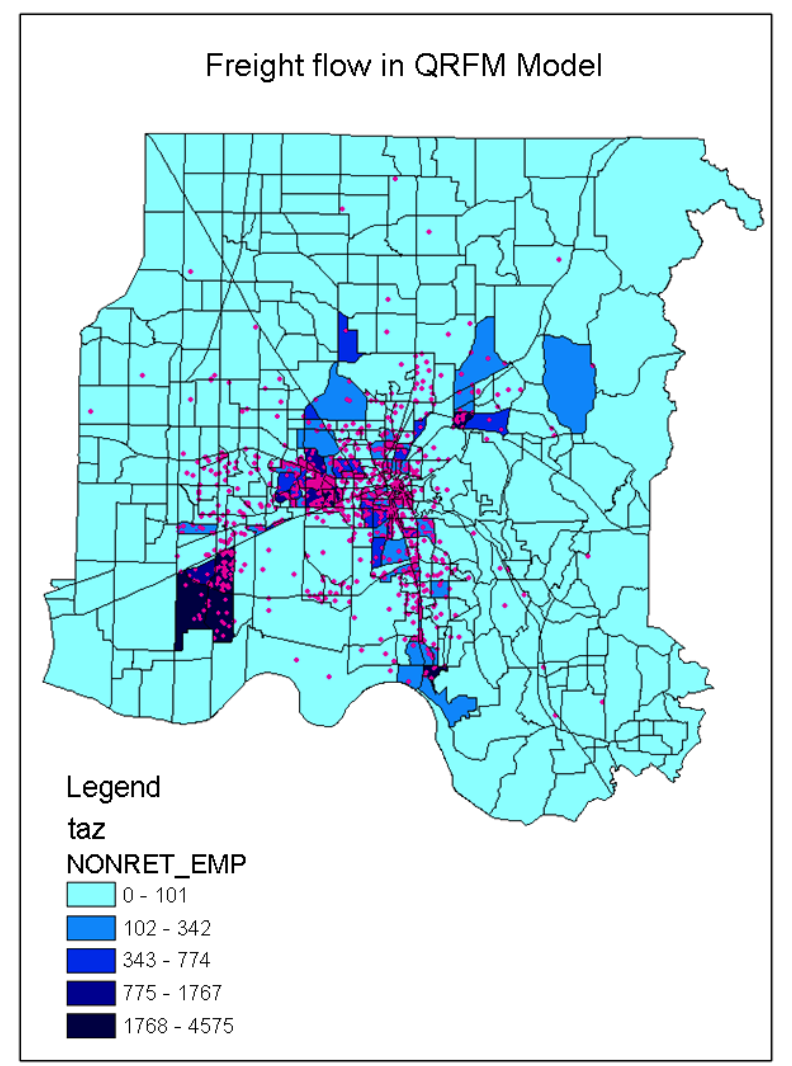

Figure 4. Freight trips versus non-retail employment.

upon development of a successful application. Though not used, the analysis could have benefitted by employing dynamic traffic assignment techniques, such as those available in PARAMICS, VISSUM or VISTA, that have the capability to move vehicles through the network using car following and lane changing models [5].

\section{Statistical Analysis}

An analysis of the model for calculating truck trips was performed by developing freight trip purposes and designing a series of travel modules to perform trip distribution plus assigning freight trips to roadways in the network. Initially, the trips produced and attracted were distributed using a gravity model that treated truck trips similar to how passenger trips are treated by distributing freight trips to zones within the study area. Truck counts at external stations in the model were included as a separate trip purpose and distributed between these stations. For traffic assignment, the freight trips were assigned to the network without the presence of passenger cars using the shortest path algorithm where all trucks were assumed to take the shortest travel time path through a network. This algorithm limits the number of trucks assigned to local roadways due to the slow travel speeds on these roadways, a result that could also have been obtained with an impedance function. Though the possibil- 
ity exists for some trucks to be assigned to local roadways, the number of such trucks is assumed to be minimal.

The accuracy of the assignment of truck volumes was determined by comparing the assignment results to the actual truck volumes reported by Alabama Department of Transportation (ALDOT). The first comparison used a scatter plot of actual truck traffic volume versus traffic volumes from the QRFM. That plot in Figure 5 shows that there is no clear relationship between the QRFM results and the actual freight counts in Huntsville using the $100 \%$ internal distribution.

To statistically measure the difference between the assigned truck traffic and actual truck counts, the Nash-
Sutcliffe (NS) coefficient was calculated [6]. The value of this coefficient ranges from $-\infty$ to 1 , with a coefficient of one $(E=1)$ showing a perfect match of forecasted counts to the ground counts. A zero coefficient $(E=0)$ shows that the forecasted values are as accurate as the mean of the ground counts, whereas a coefficient less than zero $(-\infty<E<0)$ occurs when the forecasted mean is less than the ground counts. In other words, this coefficient gives a measure of scatter variation from the 1:1 slope line of modeled truck counts versus the ground counts. The more deviation of points from the 1:1 slope line, the lower the coefficient. The greater the NS-value the better is the forecast. This coefficient can be calculated using the formula:

$$
\mathrm{NS}=1-\sum_{1}^{\mathrm{n}}(\text { Modeled Counts }- \text { Ground Counts }) / \sum_{1}^{\mathrm{n}}(\text { Ground Counts }- \text { Mean Ground Counts })^{2}
$$

The application of the Nash-Sutcliffe test to the data gave an efficiency coefficient of -1.45 showing that taking an average value of the truck counts from ALDOT would better predict truck flows than the travel demand model.

Further statistical tests were performed to determine whether the data obtained from the travel demand model were similar to actual truck counts. The MINITAB ${ }^{\text {TM }}$ statistical software was used to conduct the analysis of variance (ANOVA) test. The results provide statistical evidence to suggest that actual truck volumes are different from the volumes assigned by the model.

To improve the results, an alternate trip distribution method was employed. This method was developed from the results of a study being done in Mobile, Alabama [7]. The flow patterns collected from the Mobile area in Table 1 show that external-internal (E-I) truck trips and internal-external (I-E) truck trips represent over $80 \%$ of

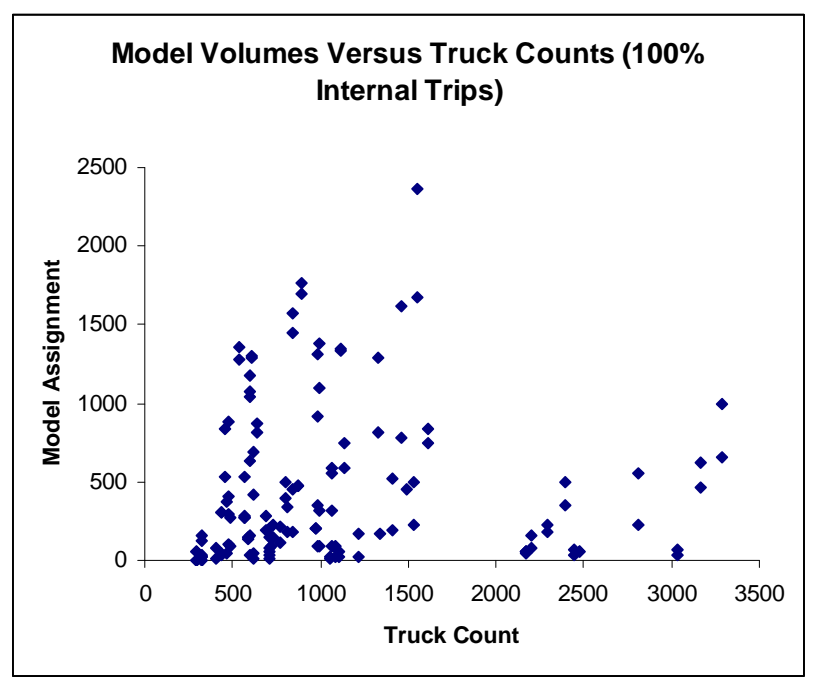

Figure 5. Scatter plot of truck traffic. the total truck volumes, while the internal-internal (I-I) truck trips account for less than 20\%. This implies that approximately $80 \%$ of the raw materials for manufacturing are generated outside the area, and approximately $80 \%$ of the finished products are exported to points outside the area.

To account for changes in truck distribution in the model, the modules used to run the Huntsville MPO travel demand model were adjusted to account for freight trips distributed into the community from outside (E-I), and outward from the community to points beyond the study area (I-E). An experiment was designed to include four different trip distribution levels:

- $90 \%$ (E-I and I-E) and 10\% (I-I),

- $80 \%$ (E-I and I-E) and 20\% (I-I),

- $70 \%$ (E-I and I-E) and 30\% (I-I), and

- $60 \%$ (E-I and I-E) and 40\% (I-I).

The reason for not simply using the $80 \%$ E-I and I-E found in the Mobile project is the uncertainty that Huntsville would perform similarly as Mobile due to socioeconomic differences in the two communities and the influence of the Port of Mobile.

The E-I and I-E truck trip distributions were developed using the total number of trucks crossing the study area boundary. The total number of trucks at the boundaries was split by percentage into the number of trucks expected to enter and leave the community (E-I and I-E)

Table 1. Freight locations for mobile area.

\begin{tabular}{ccc}
\hline $\begin{array}{c}\text { Freight Origin/Destination } \\
\text { Location }\end{array}$ & Origins & Destinations \\
\hline Within Mobile County & $14.5 \%$ & $16.4 \%$ \\
Outside Mobile County & $84.5 \%$ & $80.7 \%$ \\
Local Port & $1.0 \%$ & $2.8 \%$ \\
\hline
\end{tabular}


and the number of trucks passing through the community. Parameters in the gravity model were set to constrain the E-I and I-E truck numbers such that the total number of trucks at the external stations did not exceed boundary conditions. A separate gravity model, which used the modeling details for the City of Huntsville, was used for the internal truck trips that included a reduction factor to limit the number of trips. As before, mode split was not included in the model and truck trips were assigned to the Huntsville network without passenger cars to allow truck access to the major roadways.

A scatter plot was drawn to compare actual truck count and the trucks assigned from the model for each percentage split. A scatter plot for the $80 \%$ E-I and I-E with $20 \%$ internal trips is shown in Figure 6. As can be seen, the results appear to align much closer to the 1:1 slope with the trip distribution adjustment.

For comparison, the Nash-Sutcliffe efficiency coefficient was calculated for each trip distribution split. The results were as follow:

- NS Coefficient $=0.59$ for the $90 \%$ (E-I and I-E) and $10 \%$ (I-I),

- $\quad$ NS Coefficient $=0.61$ for the $80 \%$ (E-I and I-E) and $20 \%$ (I-I),

- $\quad$ NS Coefficient $=0.62$ for the $70 \%$ (E-I and I-E) and $30 \%(\mathrm{I}-\mathrm{I})$, and

- $\quad$ NS Coefficient $=0.61$ for the $60 \%$ percent (E-I and I-E) and 40\% (I-I).

As these results show, there is little difference between the models. However, all the models show significant improvements over the $100 \%$ internal distribution.

Further statistical tests were performed to determine if the data obtained from the travel demand model were similar to actual truck counts. MINITAB ${ }^{\text {TM }}$ Statistical Software was used to analyze the data and to perform the analysis of variance (ANOVA) test. The results show no statistical evidence that actual truck volumes are different from the volumes assigned by the model. Further, using

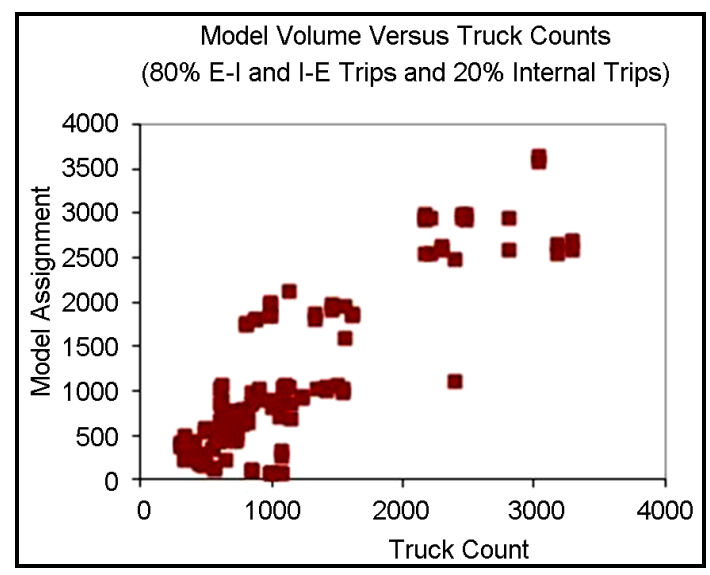

Figure 6. Scatter plot of truck traffic with distribution modification. the Mann-Whitney non-parametric test, it is found that that the QRFM data likely come from the same population as the actual data.

The implementation of the methodology would require that smaller communities develop a truck trip purpose using the QRFM equations. However, the resulting truck values need to be converted into internal truck trips and internal-external and external-internal truck trips based on the percent trips expected to leave the study area. Then, the two purposes need to be included into the modeling process as would be followed using any number of trip purposes.

\section{Statistical Analysis}

This paper demonstrated that trip generation equations from the QRFM, when calculated using socio-economic data from a medium sized travel demand model, can accurately reflect the locations where truck trips are likely to originate or terminate inside a community. Secondarily, this paper showed that the use of an appropriate trip distribution method that accounts for freight movements entering and leaving the study area produces an accurate forecast of trucks on existing roadway infrastructure, the percent values to use will be based on varying the data and determining the best fit or using the recommended values presented in this paper. This ability to successfully model freight in an urban area can be used to overcome the limitation of neglecting freight in travel demand modeling processes.

\section{REFERENCES}

[1] Cambridge Systematics, Inc., "Quick Response Freight Manual," Federal Highway Administration, Washington DC, 1996.

[2] Cambridge Systematics, Inc., "Quick Response Freight Manual II," Federal Highway Administration, Washington DC, 2007.

[3] J. de Dois Ortuzar and L. G. Willumsen, "Modeling Transport," 2nd Edition, John Wiley and Sons, New York, 1994.

[4] Huntsville MPO (Metropolitan Planning Organization), "Information Provided by Mr. James Moore, Transportation Planner for Huntsville," AL Metropolitan Planning Organization, Decatur, 2007.

[5] M. Jeihani, "A Review of Dynamic Traffic Assignment Computer Packages," Journal of the Transportation Research Forum, Vol. 46, No. 2, 2007, pp. 35-46.

[6] J. E. Nash and J. V. Sutcliffe, "River Flow Forecasting Through Conceptual Models Part I: A Discussion of Principles." Journal of Hydrology, Vol. 10, No. 3, 1970, pp. 282-290. doi:10.1016/0022-1694(70)90255-6

[7] J. S. Thompson, K. E. Yarbrough, M. D. Anderson, G. A. Harris and K. Harrison, "An Approach to Collecting Local Freight Information," Transportation Research Board, Washington DC, 2010. 